\title{
Dual-specificity histone demethylase KIAA1718 (KDM7A) regulates neural differentiation through FGF4
}

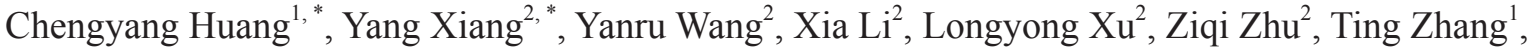 \\ Qingqing Zhu ${ }^{1}$, Kejing Zhang ${ }^{1}$, Naihe Jing ${ }^{1}$, Charlie Degui Chen ${ }^{2}$ \\ ${ }^{1}$ Laboratory of Molecular Cell Biology, ${ }^{2}$ State Key Laboratory of Molecular Biology, Shanghai Key Laboratory of Molecular An- \\ drology, Institute of Biochemistry and Cell Biology, Shanghai Institutes for Biological Sciences, Chinese Academy of Sciences, 320 \\ Yueyang Road, Shanghai 200031, China
}

Dimethylations of histone $\mathrm{H3}$ lysine 9 and lysine 27 are important epigenetic marks associated with transcription repression. Here, we identified KIAA1718 (KDM7A) as a novel histone demethylase specific for these two repressing marks. Using mouse embryonic stem cells, we demonstrated that KIAA1718 expression increased at the early phase of neural differentiation. Knockdown of the gene blocked neural differentiation and the effect was rescued by the wild-type human gene, and not by a catalytically inactive mutant. In addition, overexpression of KIAA1718 accelerated neural differentiation. We provide the evidence that the pro-neural differentiation effect of KDM7A is mediated through direct transcriptional activation of FGF4, a signal molecule implicated in neural differentiation. Thus, our study identified a dual-specificity histone demethylase that regulates neural differentiation through FGF4.

Keywords: histone demethylase, KIAA1718, KDM7A, neural differentiation, FGF4

Cell Research (2010) 20:154-165. doi: 10.1038/cr.2010.5; published online 19 January 2010

\section{Introduction}

In eukaryotic cells, DNA is associated with histones to form the nucleosome, the building block of chromatin. The nucleosome is composed of two copies each of the four core histone proteins $\mathrm{H} 2 \mathrm{~A}, \mathrm{H} 2 \mathrm{~B}, \mathrm{H} 3$, and $\mathrm{H} 4$, wrapped by 146 base pairs of DNA [1]. Histone proteins are subjected to a variety of posttranslational modifications, including acetylation, phosphorylation, methylation, ubiquitination, and ADP-ribosylation [2, 3], which either alone or in combination, contribute to the epigenetic program and are referred to as the 'histone code' [4].

Histone $\mathrm{H} 3$ lysine 9 dimethylation (H3K9me2) is an important posttranslational modification associated with transcriptional silencing. In contrast to $\mathrm{H} 3 \mathrm{~K} 9 \mathrm{me} 3$ that is localized in pericentric heterochromatin, $\mathrm{H} 3 \mathrm{~K} 9 \mathrm{me} 2$ is

\footnotetext{
*These two authors contributed equally to this work.

Correspondence: Charlie Degui Chen ${ }^{\mathrm{a}}$, Naihe Jing ${ }^{\mathrm{b}}$

${ }^{a}$ Tel: 021-54921148

E-mail: cdchen@sibs.ac.cn

${ }^{b}$ E-mail: njing@sibs.ac.cn

Received 29 April 2009; revised 26 November 2009; accepted 2 December 2009; published online 19 January 2010
}

mainly localized within the silent regions of the euchromatin [5-8]. At least three methyltransferases, G9a, GLP1 (EuHMTare), and ESET (SetDB1), have been identified to be able to catalyze the formation of $\mathrm{H} 3 \mathrm{~K} 9 \mathrm{me} 2$ [7, 9-12]. G9a is the major histone methyltransferase for H3K9me2 formation, because knockout of this gene in mice causes a $90 \%$ loss of $\mathrm{H} 3 \mathrm{~K} 9 \mathrm{me} 2[5,6,8]$. Knockout of this gene resulted in embryonic lethality and the embryos failed to develop past E8.5. Although GLP-1 (EuHMTare) itself is also a methyltransferase for the formation of H3K9me2 [10], it forms a heteromeric complex with G9a and its knockout phenocopied G9a deletion [10]. ESET (SetDB1) is also able to catalyze H3K9me2 formation in euchromatin and its knockout resulted in periimplantation lethality between 3.5 and $5.5 \mathrm{dpc}$ [9, 11-13]. The knockout studies indicate that the level of H3K9me2 has to be maintained for proper embryonic development.

Histone H3 lysine 27 dimethylation (H3K27me2) is also a repressive mark associated with transcriptional silencing [14]. The Polycomb repressive complex 2 (PRC2) that contains Ezh2, Eed, and Suz12 catalyzes the formation of this mark $[15,16]$. The three components of the PRC2 complex are all essential for embryonic development, since knockout of any one of them leads to severe 
defects during gastrulation [17-19].

Similar to other posttranslational modifications, histone methylation is reversible. Histone methylation can be reversed by histone demethylases. LSD1 is the first histone demethylase identified, which can remove di- and mono-methylation from $\mathrm{H} 3 \mathrm{~K} 4$ using an amine oxidase reaction [20]. Subsequently, a JmjC domain-containing protein was identified to possess histone demethylase activity, and the JmjC domain was shown as a demethylase signature motif [21]. This class of enzymes catalyzes the demethylation by a hydroxylation reaction and requires both iron and $\alpha$-ketoglutarate as cofactors.

Previously, we and others identified $30 \mathrm{JmjC}$ domaincontaining proteins in humans through analysis of public protein-domain databases [22-24]. These proteins can be classified into seven groups based on the domain structure of the full-length protein. Five of the seven groups have been demonstrated to be histone lysine demethylases [25-37]. Although proteins in the family of PHF2/ PHF 8 contain conserved residues for cofactor binding, the biochemical functions of the proteins in the family were still unknown.

In this report, we show that KIAA1718, a member of the PHF2/PHF8 family, is a histone demethylase specific for both $\mathrm{H} 3 \mathrm{~K} 9 \mathrm{me} 2$ and $\mathrm{H} 3 \mathrm{~K} 27 \mathrm{me} 2$. Using a mouse embryonic stem cell (ESC) differentiation system, we demonstrate that KIAA1718 plays an important role in neural differentiation. The neural promoting effect is mediated by FGF4, a molecule involved in neural differentiation. Thus, our study identified a novel histone demethylase for $\mathrm{H} 3 \mathrm{~K} 9 \mathrm{me} 2$ and $\mathrm{H} 3 \mathrm{~K} 27 \mathrm{me} 2$ that plays an important role in neural differentiation.

\section{Results}

\section{KIAA1718 is a histone demethylase}

KIAA1718 is a member of the PHF2/PHF8 family of the $\mathrm{JmjC}$ domain-containing proteins and contains two recognizable domains: a PHD and a JmjC domain (Figure 1A). To determine whether KIAA1718 has histone demethylase activity, we expressed and purified fulllength His-tagged protein from baculovirus-infected insect cells (Figure 1B). The protein was subjected to an in vitro assay with bulk histones, and immunoblotting was used to screen for its substrates. As shown in Figure 1, KIAA1718 had demethylase activity for H3K9me2 and, a weaker activity but with a similar kinetics, for H3K27me2 (Figure 1C and 1D). By contrast, H3K9me1, $\mathrm{H} 3 \mathrm{~K} 9 \mathrm{me} 3, \mathrm{H} 3 \mathrm{~K} 27 \mathrm{me}$, and H3K27me3 were not affected, nor were mono-, di-, or trimethylated H3K4, H3K36, H3K79, and H4K20 (Figure 1C). MALDI-TOF mass spectrometric analysis revealed that KIAA1718 con- verted both $\mathrm{H} 3 \mathrm{~K} 9 \mathrm{me} 2$ and $\mathrm{H} 3 \mathrm{~K} 27 \mathrm{me} 2$ to monomethyl and nonmethyl peptides, but with weak, if at all, activity against H3K9me1 and H3K27me1 (Figure 1E). Demethylation by KIAA1718 requires its JmjC domain, because mutation of a conserved histidine (H281A) in the JmjC domain abolished the activity (Figure 1F). These data suggest that KIAA1718 is a histone demethylase specific for $\mathrm{H} 3 \mathrm{~K} 9 \mathrm{me} 2$ and $\mathrm{H} 3 \mathrm{~K} 27 \mathrm{me} 2$ in vitro.

\section{KIAA1718 plays an important role in neural differentia-} tion

Three methyltransferases are able to methylate H3K9 to form H3K9me2. They are ESET/SETDB1, G9a, and Eu-HMTase/GLP-1. Knockout of any one of them resulted in embryonic lethality from E3.5 to E9.5 [8, 10, 13]. Similarly, H3K $27 \mathrm{me} 2$ formation is catalyzed by the PRC2 complex that contains Ezh2, Eed, and Suz12, and knockout of any one of them led to severe defects during gastrulation [17-19]. These data suggest that the proper levels of $\mathrm{H} 3 \mathrm{~K} 9 \mathrm{me} 2$ and $\mathrm{H} 3 \mathrm{~K} 27 \mathrm{me} 2$ are essential for cell differentiation during embryonic development, and a demethylase for these two modifications may also play key roles in this process.

We thus tested whether KIAA1718 is involved in differentiation of ESCs. We established short hairpin RNA (shRNA)-mediated KIAA1718 knockdown ESCs by lentiviral transduction (Figure 2A). Both shRNA1 and shRNA2 knocked down about $85 \%$ of KIAA1718 expression and shRNA3 did not show any knockdown effect. Using an unbiased differentiation method (hanging drop), we discovered that KIAA1718 knockdown mainly blocked neural differentiation, since the levels of the neural precursor markers Sox 1 and Nestin were strongly reduced (Figure 2B). Consistently, the levels of pluripotency markers Oct4 (also known as Pou5f1), Nanog, and Rex 1 were elevated. However, the markers for endoderm (Gata4 and Gata6) and mesoderm (Flk1 and Brachyury) were not significantly altered. We also compared the gene expression profiles between control and knockdown cells. Among 45038 probes, 899 probes were downregulated and 650 probes were upregulated after KIAA1718 knockdown. Gene Ontology analysis revealed that a significant portion of the regulated genes was in development hierarchies, and nervous system development was most significant within the system development category (Supplementary information, Figure S1). These results suggest that KIAA1718 is mainly involved in neural differentiation.

To further test this, we induced ESC differentiation by a neural differentiation protocol that can normally achieve about $80 \%$ neural differentiation. Expression analysis revealed that, among 24 genes encoding JmjC 
A

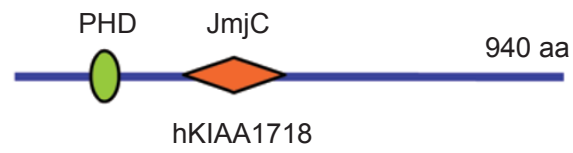

C
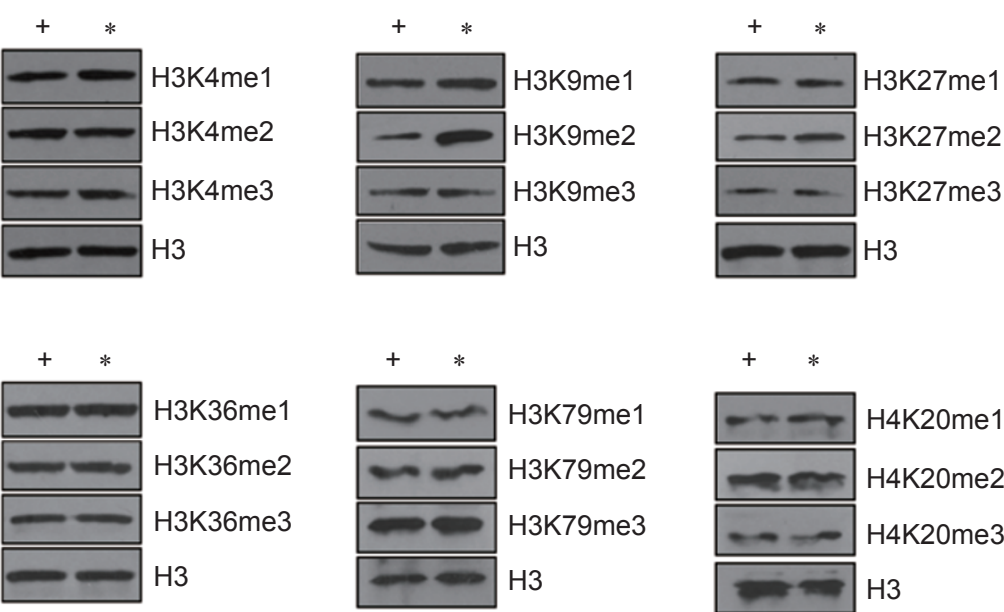

B

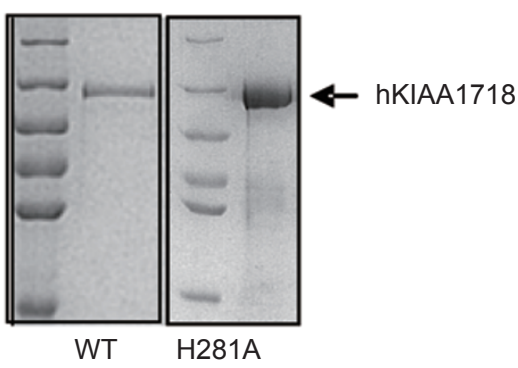

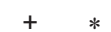

D

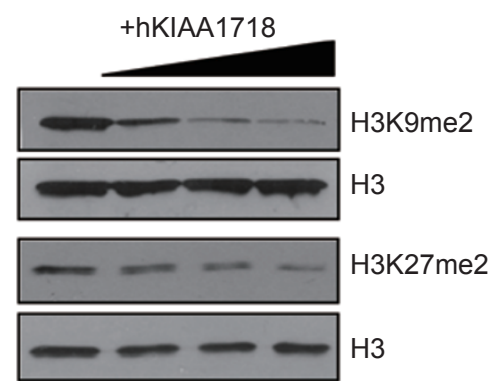

F Wt H281A

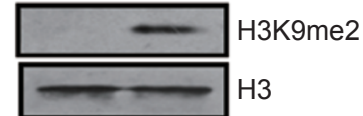

+ Native

* Heat inactivated
H3K9me2

2749.6

H3K27me2

2944.2

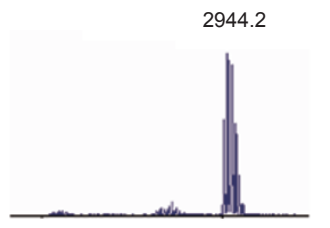

No enzyme
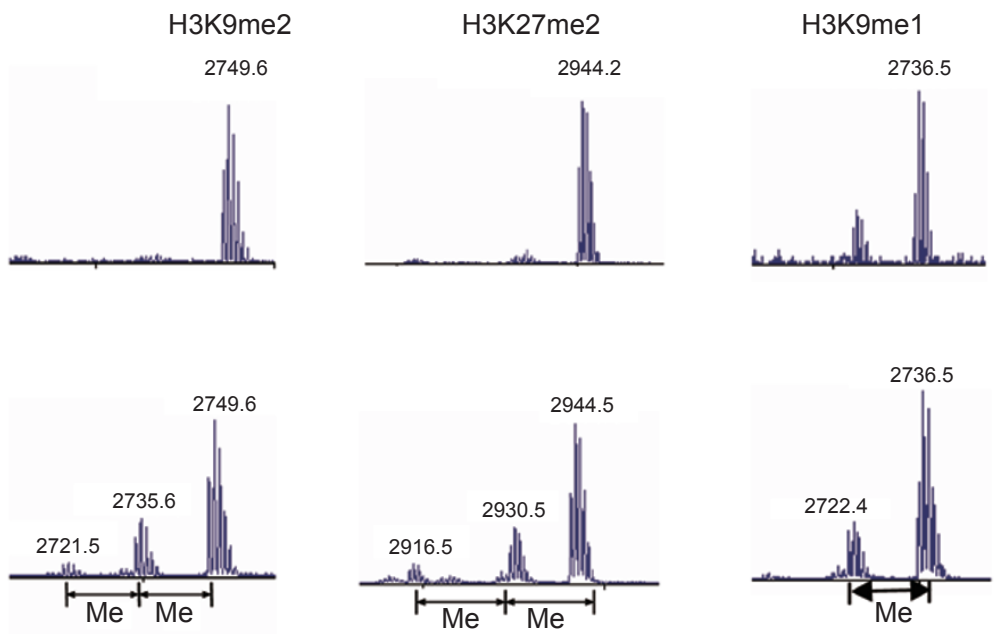

H3K27me1

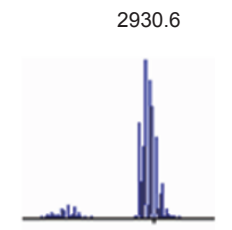

hKIAA1718

Figure 1 KIAA1718 is a histone demethylase. (A) The domain structure of human KIAA1718. (B) Purified His-tagged recombinant wild-type and mutant KIAA1718 separated by SDS-PAGE and stained by Coomassie blue. (C and D) Calf thymus histones were reacted with the native or heat-inactivated recombinant KIAA1718 protein (C), or increasing doses of KIAA1718 protein $(0,2,4$ and $8 \mu \mathrm{g})(\mathrm{D})$, and assayed by immunoblotting. (E) Mass spectrometry of demethylation reaction using various peptides. Numbers refer to $\mathrm{m} / \mathrm{z}$ values. (F) Immunoblotting of in vitro demethylation assay in which calf thymus histones were reacted with the $\mathrm{H} 281 \mathrm{~A}$ mutant $(\mathrm{H} 281 \mathrm{~A})$. 
A

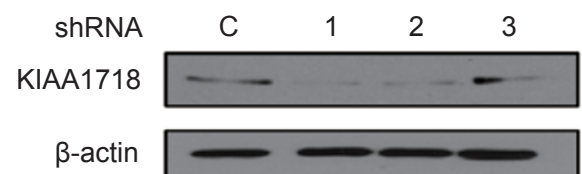

C

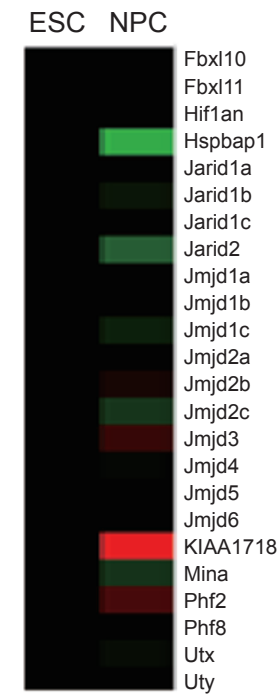

$-3$

$+3$

F.C. $\left(\log _{2}\right)$
B

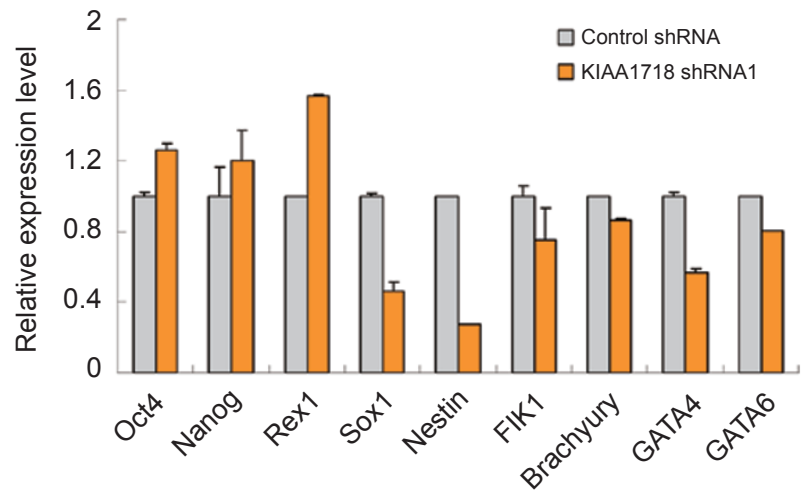

D

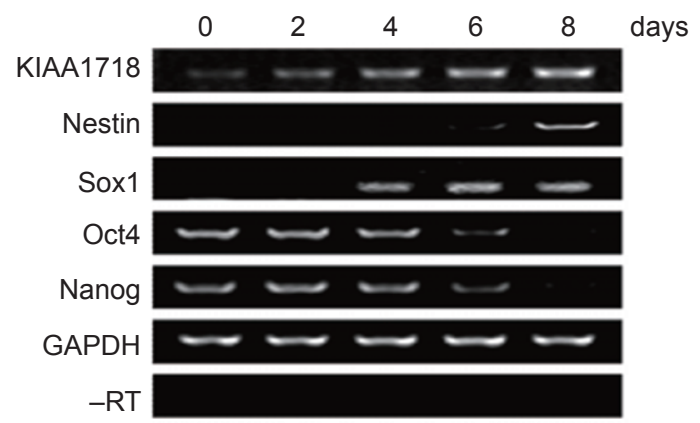

E

G

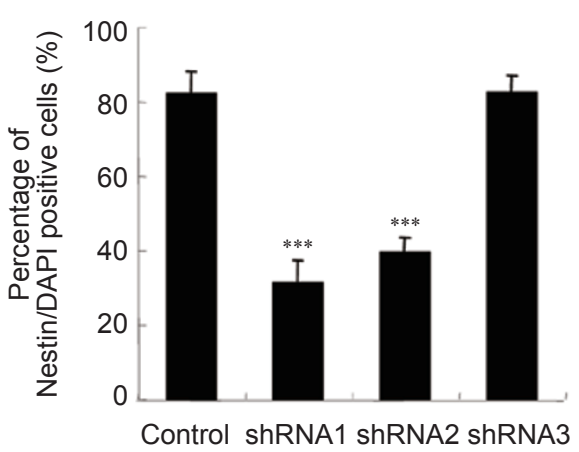

Figure 2 KIAA1718 positively regulates ESC neural differentiation. (A) Western blot analyses of KIAA1718 expression in control and shRNA-mediated knockdown of ESCs. shRNA1 and shRNA2 had a knockdown efficiency of $80 \%-90 \%$, but control and shRNA3 did not. (B) Quantitative RT-PCR analysis of different markers after ESC differentiation by hanging drop experiments. (C) Expression change of JmjC domain-containing proteins between ESCs and NPCs by expression profiling. Data are expressed as fold change (F.C.). (D) RT-PCR analysis of different mRNA levels during 8 days of neural differentiation. (E) Immunoblotting of KIAA1718 protein during ESC neural differentiation. (F and G) Immunofluorescent staining and quantification of Nestin-positive cells after 8 days of neural differentiation from ESCs with control or KIAA1718 shRNAs. ${ }^{* * *} P<0.01$. 
domain-containing proteins in the Affymetrix microarray, KIAA1718 was the most highly induced one after 8 days of differentiation of wild-type ESCs to neural precursor cells (NPCs) (Figure 2C). Real-time PCR and immunoblotting confirmed that expression of KIAA1718 was increased during this process (Figure 2D and 2E). These data support our hypothesis that KIAA1718 is involved in neural differentiation. To test this further, we compared neural differentiation between the wildtype and KIAA1718 knockdown ESCs. After 8 days of neural differentiation, ESCs expressing control shRNA or KIAA1718 shRNA3, in which KIAA1718 expression was not inhibited (Figure 2A), generated about $80 \%$ Nestin-positive NPCs, whereas ESCs expressing inhibitory shRNA1 or shRNA2 exhibited impaired neural differentiation, with only $30-40 \%$ Nestin-positive NPCs and weaker Nestin signals (Figure 2F and 2G). Consistently, the number of Tuj1- and MAP2-positive neuronal cells was decreased in KIAA1718 knockdown cells (Supplementary information, Figure S2).

To confirm our conclusion, we performed rescue experiments. Because R1 ES cells are of mouse origin, we forced the expression of human KIAA1718 in shRNA1 knockdown cells by lentiviral transduction. The virus vector expressing a red fluorescent protein (RFP) was used for cell sorting. Western blot analysis demonstrated that both the wild-type human gene and the catalytically inactive mutant escaped the knockdown of shRNA1. These two types of cells expressed a similar level of total KIAA1718 as the parental control cells (Figure $3 \mathrm{~A})$. Forced expression of the wild-type hKIAA1718 led to significant rescue of neural differentiation, but the rescue effect was greatly compromised when the catalytically inactive mutant was expressed (Figure 3B and $3 \mathrm{C})$. These data confirm that KIAA1718 is necessary for neural differentiation and that the function of histone demethylase is required for this process.

We also used ES cell lines stably overexpressing wildtype and the catalytically inactive H281A mutant of KIAA1718 to examine its role in neural differentiation. Western blot analysis demonstrated that both the wildtype human gene and the inactive mutant had a similar level of overexpression (Figure 4A). Overexpression of wild-type KIAA1718, but not the catalytically inactive mutant, accelerated neural differentiation, as demonstrated by the appearance of more Nestin-positive NPCs in the wild-type KIAA1718 group than in the control and H281A mutant groups (Figure 4B and 4C). KIAA1718 overexpression did not affect the expression of Oct 4 in ESCs, indicating that the cells maintained their undifferentiated state (Figure 4D and 4E). In addition, the levels of mesoderm markers (Brachyury, Flk1) and endoderm
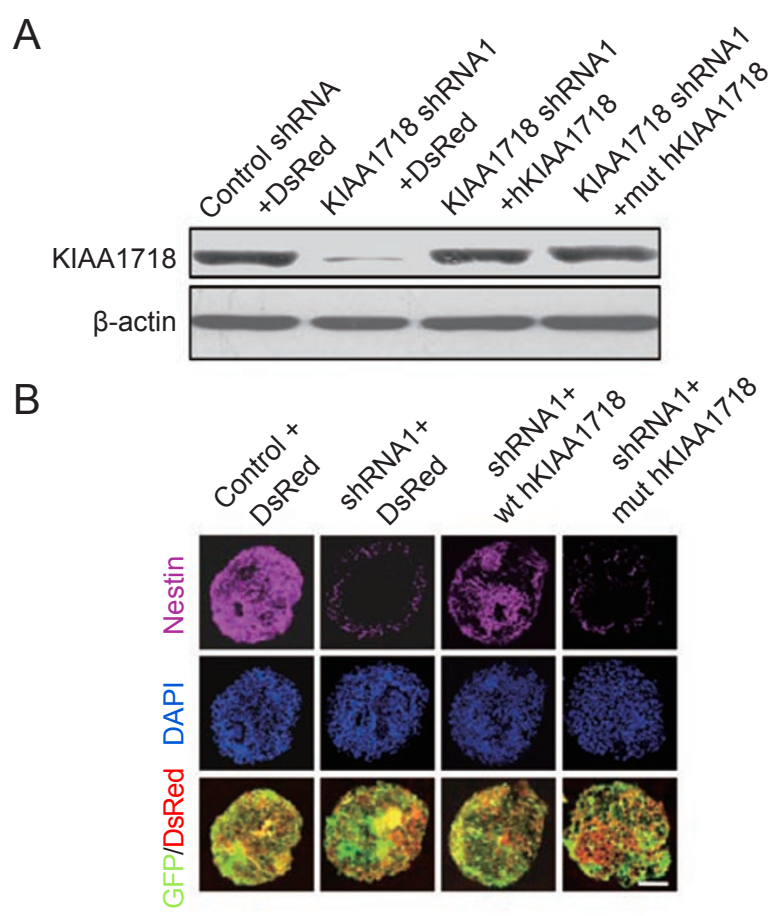

C

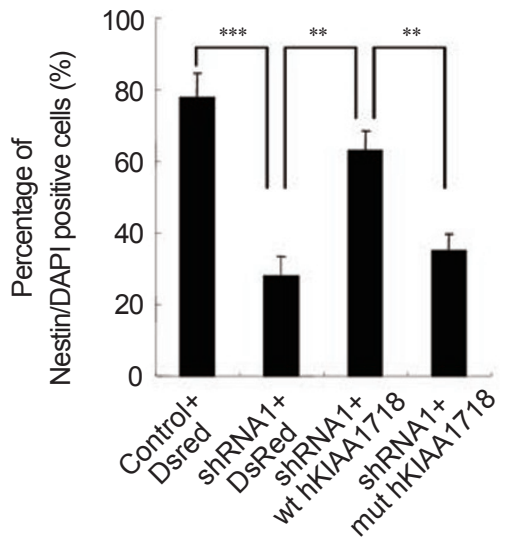

Figure 3 Neural differentiation defects by KIAA1718 knockdown was rescued by human protein. (A) Immunoblotting of total KIAA1718 protein levels from ESCs with control or KIAA1718 shRNA1, or shRNA1 knockdown of ESCs expressing human wild-type KIAA1718 (hKIAA1718) or catalytically inactive H281A mutant (mut hKIAA1718). The vectors carry RFP that was used for cell sorting. (B and $\mathbf{C}$ ) Immunofluorescent staining and quantification of Nestin-positive cells after 8 days of neural differentiation from ESCs with control, KIAA1718 shRNA1, KIAA1718 shRNA1 knockdown expressing human wild-type KIAA1718 (hKIAA1718), or catalytically inactive H281A mutant (mut hKIAA1718). $* * P<0.01, * * * P<0.005$. Scale bar $=20 \mu \mathrm{m}$.

markers (GATA4, GATA6) were not changed between the control and KIAA1718 overexpressed ESCs (Figure 4F). These data suggest that KIAA1718 overexpression specifically facilitates ESC neural differentiation. 
A

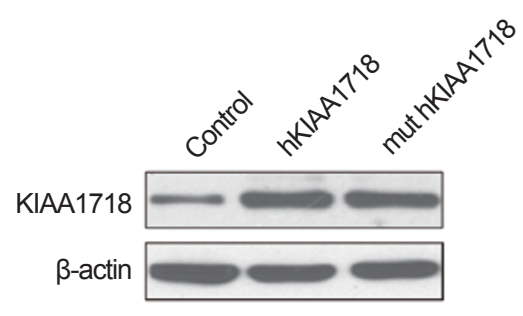

C

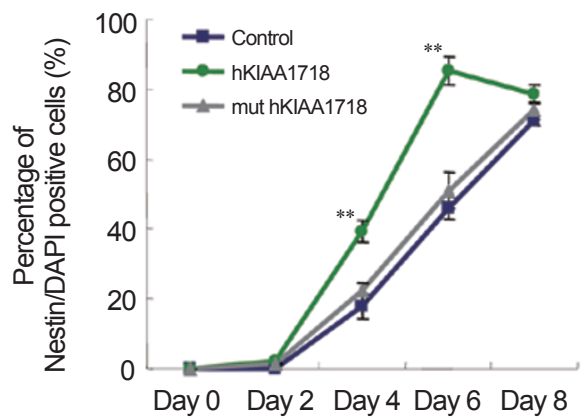

B

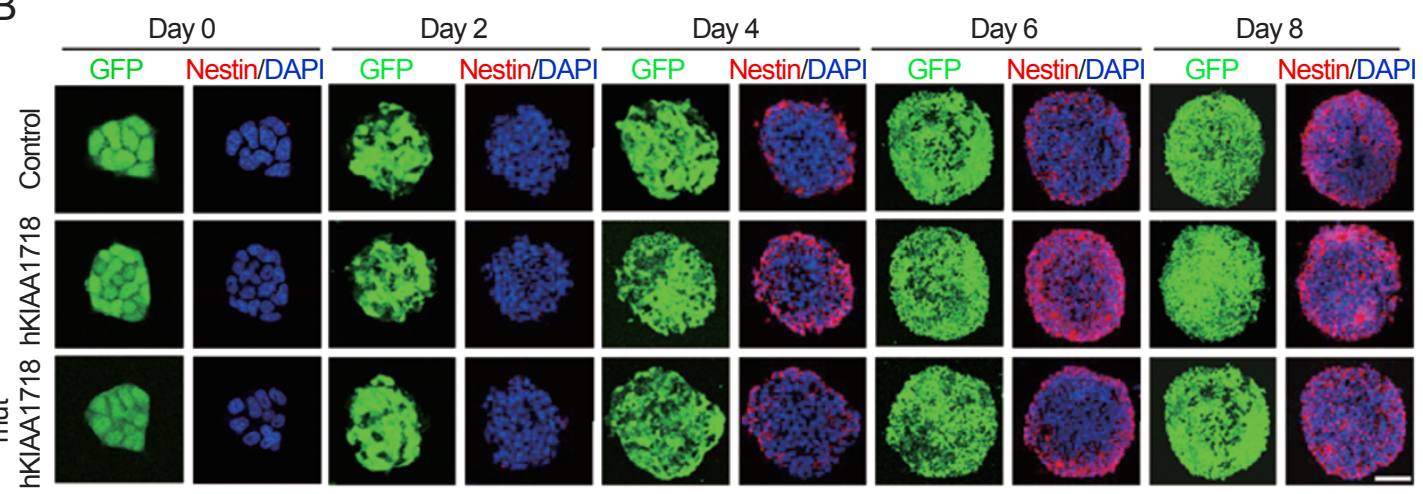

D

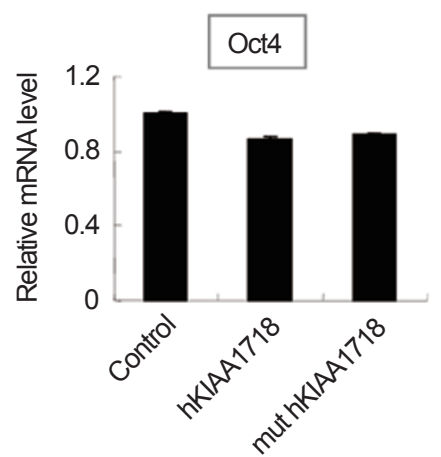

E

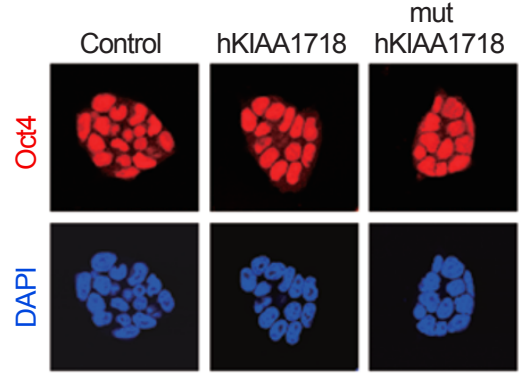

F
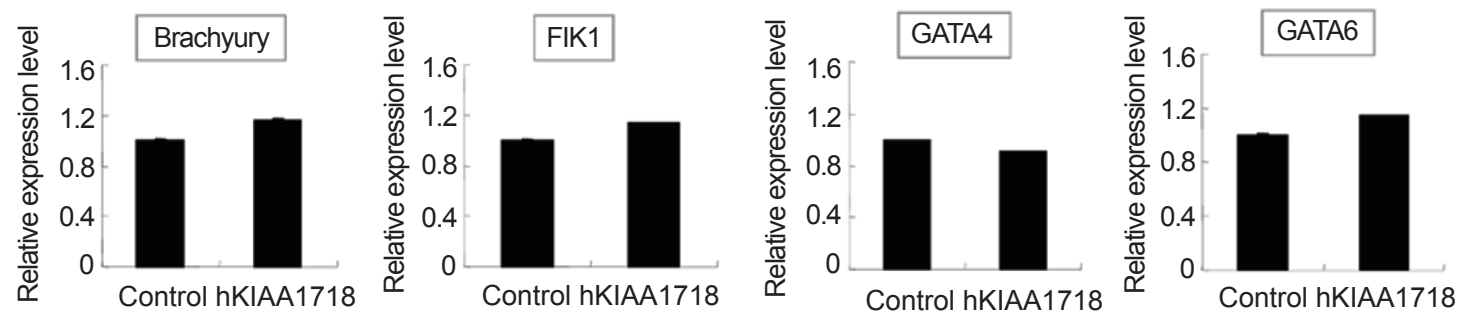

Figure 4 KIAA1718 overexpression accelerated neural differentiation in ESCs. (A) Western blot analyses of total KIAA1718 protein levels in ESCs stably overexpressing human wild-type KIAA1718 or H281A mutant. (B) Immunostaining of NPC marker Nestin in the course of neural differentiation for control, wild-type, and mutated human KIAA1718 overexpressed ESCs. GFP is co-expressed for sorting. (C) Quantification of Nestin-positive cells in the course of neural differentiation from ESCs overexpressing hKIAA1718 or mut hKIAA1718. Data are from three independent experiments. ${ }^{* *} P<0.01$. (D and E) Q-PCR analysis of Oct4 mRNA level and Oct4 immunostaining in KIAA1718 overexpressed ESCs. (F) Quantitative PCR analysis of mesoderm (Brachyury and FIk1) and endoderm (Gata4 and Gata6) markers in the control and KIAA1718 overexpressed ESCs after 6 days of neural differentiation. 
A
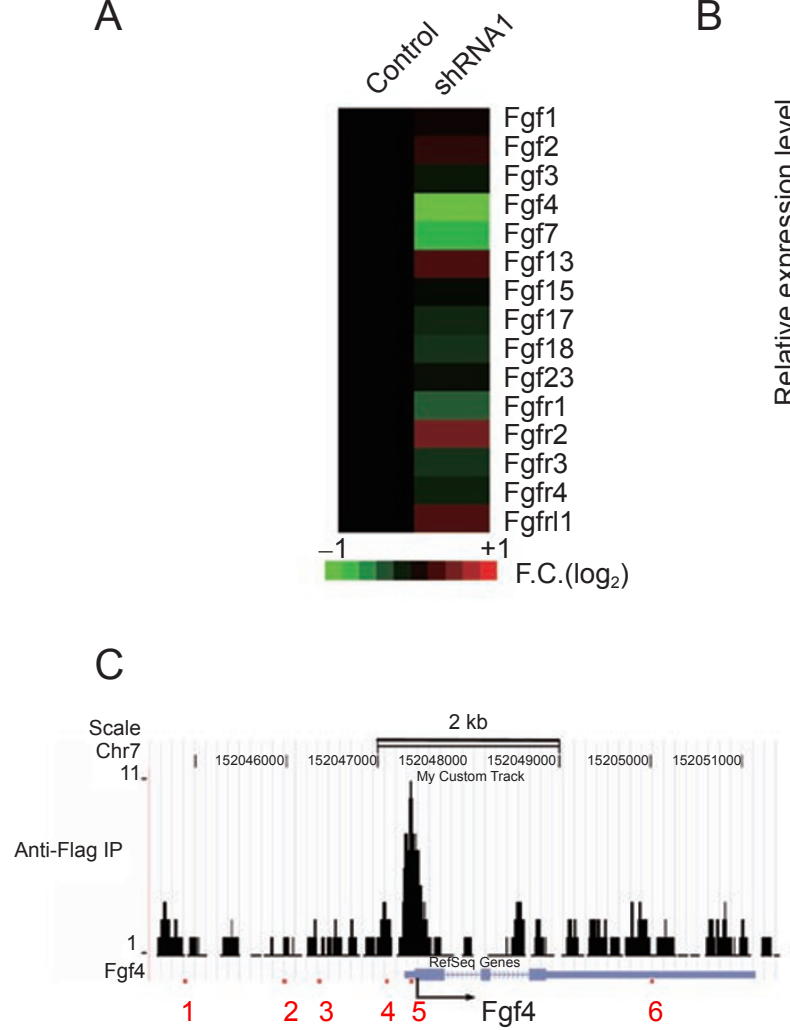

$\mathrm{D}$

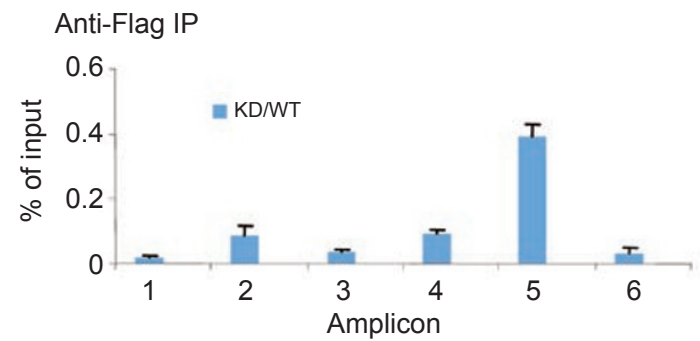

B

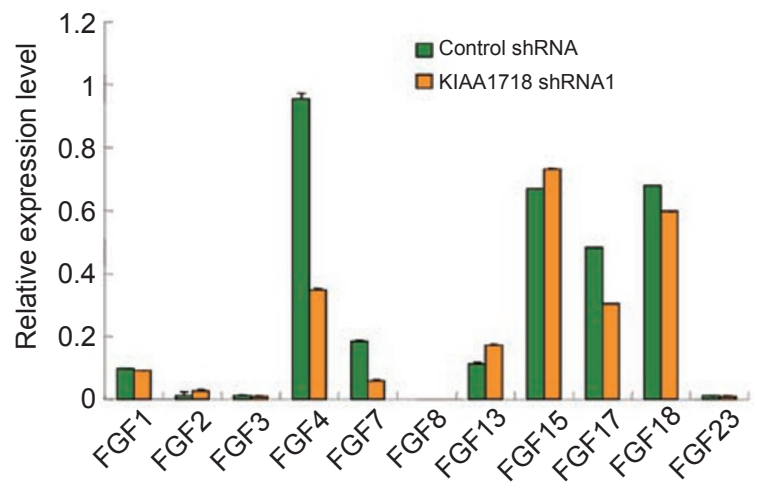

E
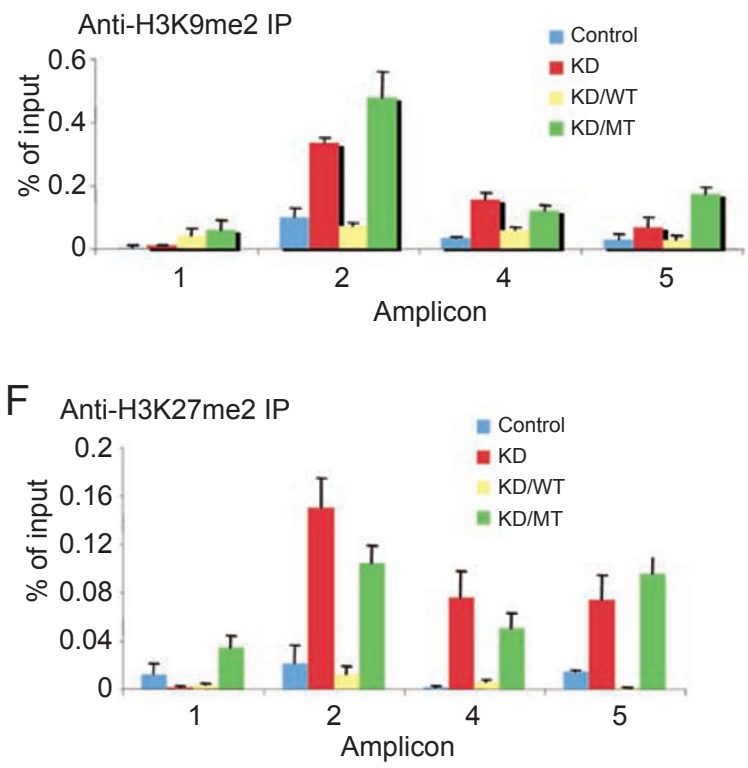

Figure 5 KIAA1718 regulates FGF signaling pathway. (A) Expression of fibroblast growth factors (FGFs) and their receptors between control and KIAA1718 knockdown ESCs using Affymetrix mouse 4302.0 microarrays. Green bars indicate downregulation and red for upregulation in KIAA1718 knockdown cells. Data are expressed as fold change (F.C.). (B) Q-PCR analysis of FGF gene expression in control and KIAA1718 knockdown ESCs. (C and D) ChIP analysis of KIAA1718-bound region in the FGF4 gene. The ChIP-Seq data and 6 amplicon locations are shown in (C) and Q-PCR results in (D). KD/wt is shRNA knocked down ESCs rescued by wild-type human Flag-hKIAA1718, and anti-flag antibody was used for precipitation (KIAA1718 antibody was not suitable for IP). (E and F) ChIP analysis of H3K9me2 (E) or H3K27me2 (F) enrichment in the promoter of FGF4 gene in ESCs of control, KIAA1718 knockdown (KD), knocked down and rescued by wild-type hKIAA1718 (KD/wt), or H281A mutant $(\mathrm{KD} / \mathrm{mt})$.

\section{KIAA1718 regulates FGF4 expression}

Neural differentiation is believed to be mediated by a default mechanism. However, multiple signaling pathways, such as FGF and BMP signals, are involved in neural induction at the gastrulation stage $[38,39]$. While FGF signaling is required for neural fate determination [40-43], BMP pathway blocks this process [41, 44, 45].
To determine whether KIAA1718 controls neural differentiation by regulating FGF signaling, we examined the expression of FGFs in KIAA1718 knockdown ESCs. Among various FGFs and FGF receptors expressed in ESCs, FGF4 was most significantly downregulated in KIAA1718 knockdown cells, as shown by microarray analysis (Figure 5A) and by real-time PCR analysis (Fig- 
A

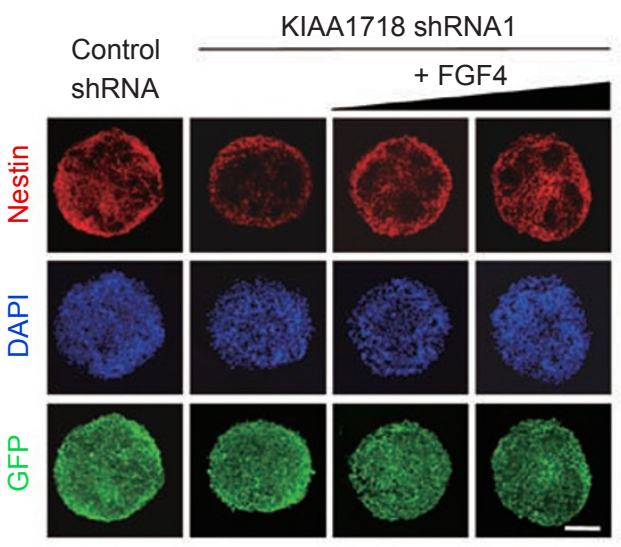

B

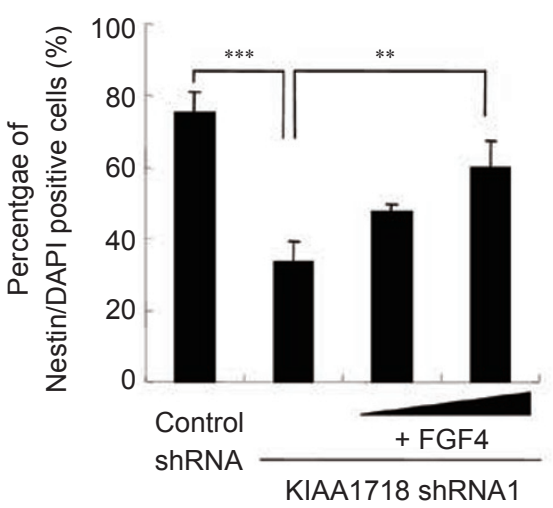

C

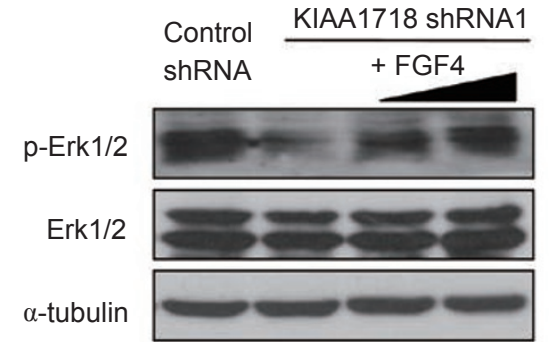

Figure 6 FGF4 rescued the defects of KIAA1718 knockdown. (A and B) Immunofluorescent staining and quantification of Nestinpositive cells after 8 days of neural differentiation from ESCs with control or KIAA1718 shRNA1 in the absence or presence of FGF4 (5 ng/ml and $15 \mathrm{ng} / \mathrm{ml})$. ${ }^{* *} P<0.01,{ }^{*} * *<0.005$. (C) Western blot analysis of Erk1/2 phosphorylation ( $p$-ERK1/2) after 1 day of neural differentiation of the ESCs with control or KIAA1718 shRNA1 in the absence or presence of FGF4 (5 ng/ $\mathrm{ml}$ and $15 \mathrm{ng} / \mathrm{ml}$ ).

ure 5B). Chromatin immunoprecipitation coupled with sequencing (ChIP-Seq) experiments using ESCs demonstrated that KIAA1718 was enriched at the promoter region of the FGF4 gene and the most enriched region (region 5) was located at the transcription start site (Figure 5C), which was confirmed by ChIP analysis. Consistent with its role as a histone demethylase for $\mathrm{H} 3 \mathrm{~K} 9 \mathrm{me} 2$ and H3K27me2, both methylation marks were greatly enriched in the KIAA1718-bound regions in KIAA1718 knockdown ESCs (Figure 5E and 5F). The increased occupancy of both marks was diminished when human wild-type KIAA1718, but not the catalytically inactive H281A mutant, was re-introduced into the cells (Figure $5 \mathrm{E}$ and $5 \mathrm{~F}$ ). These results suggest that KIAA1718 has dual-specific histone demethylase activity in vivo and directly regulates FGF4 expression.

\section{FGF4 mediates the pro-neural effect of KIAA1718}

To determine whether KIAA1718 regulates neural differentiation through FGF4, we induced neural differentiation of the KIAA1718 knockdown ESCs in the presence of FGF4. While knockdown of KIAA1718 impaired ESC neural differentiation, addition of FGF4 rescued the defects of neural differentiation in a dosedependent manner (Figure 6A and 6B). Consistently, knockdown of KIAA1718 reduced ERK1/2 phosphorylation during ESC neural differentiation, but the reduction was reversed by addition of FGF4 (Figure 6C). Since the ERK1/2 signaling pathway induced by FGF4 is critical for ESC neural differentiation [40, 42], these results indicate that KIAA1718 regulates neural differentiation through FGF4-ERK1/2 signaling.

\section{Discussion}

Although both $\mathrm{H} 3 \mathrm{~K} 9 \mathrm{me} 2$ and $\mathrm{H} 3 \mathrm{~K} 27 \mathrm{me} 2$ are important epigenetic marks associated with transcription repression and both lysine residues are flanked by identical amino acids $\left(\mathrm{ARK}_{9 / 27} \mathrm{~S}\right)$, the existence of a single demethylase capable of reversing these two marks was still an open question. Using bioinformatical and biochemical approaches, we identified KIAA1718 as a histone demethylase specific for these two well-conserved epigenetic marks. KIAA1718 belongs to a family of the JmjC domain-containing proteins whose biochemical function had not been elucidated. This group of proteins consists of PHF2, PHF8, and KIAA1718, and they share the common feature of a PHD domain and a JmjC domain. According to the proposed nomenclature for chromatinmodifying enzymes [46], KIAA1718 should be named as lysine demethylase 7A (KDM7A).

In contrast to other histone demethylases identified so far, overexpression or knockdown of KIAA1718 did not affect the global levels of H3K9me2 and H3K27me2 in several cell lines (data not shown). These data suggest that KIAA1718 probably exerts its demethylase activity 
in a gene-specific manner in vivo. Gene-specific regulation has been suggested for histone methyltransferases. For example, ESET was shown to be a methyltransferase for $\mathrm{H} 3 \mathrm{~K} 9 \mathrm{me} 2$ and $\mathrm{H} 3 \mathrm{~K} 9 \mathrm{me} 3$ formation; however, knockout of ESET in mice did not alter the global levels of these two marks [13]. Indeed, KIAA1718 regulates FGF4 expression by controlling the level of H3K9me2 and $\mathrm{H} 3 \mathrm{~K} 27 \mathrm{me} 2$ (Figure 5E and 5F).

KIAA1718 was called JHDM1D in the NCBI website because it has $45 \%$ sequence identity and $66 \%$ sequence similarity to JHDM1A and JHDM1B in the JmjC domain. Structural and biochemical studies indicate that the JmjC domain is the catalytic center for histone demethylase activity and determines the substrate specificity [47-50]. Our experiments demonstrate that KIAA1718 specifically demethylates $\mathrm{H} 3 \mathrm{~K} 9 \mathrm{me} 2$ and, with a weaker activity, H3K27me2. These results suggest that the substrate specificity of a histone demethylase may not be solely determined by the JmjC domain. The inability to predict the substrate specificity for a JmjC domaincontaining histone demethylase has been also observed for members in other families of the JmjC domain-containing proteins. For example, JHDM1B is a close homolog of JHDM1A. Both proteins share a similar overall domain structure and contain a JmjC, a PHD, a CXXC zinc-finger, and F-box domains. The sequences of their $\mathrm{JmjC}$ domains are $80 \%$ identical with the same residues required for cofactor binding. Yet, JHDM1A was shown to be a dimethyl H3K36 demethylase [21], while JHDM1B was demonstrated to be a demethylase for dimethyl H3K36 and also trimethyl H3K4 [51, 52]. More dramatically, protein sequences of the $\mathrm{JmjC}$ domains between UTX and UTY are 98\% identical; however, while UTX can remove H3K27 methylation, UTY has no histone demethylase activity at all [28]. Therefore, it is difficult to predict the substrate specificity of a histone demethylase based on the JmjC domain and if a JmjC domaincontaining protein has histone demethylase activity.

Including KIAA1718, at least eighteen histone demethylases have been identified and they can catalyze the removal of methylation from four lysine residues (H3K4, H3K9, H3K27, and H3K36) and two arginine residues (H3R2 and H4R3) [53, 54]. Similar to histone methyltransferases, demethylation of each residue may be regulated by several enzymes. For example, at least six histone demethylases have been identified as $\mathrm{H} 3 \mathrm{~K} 4$ demethylases (LSD1, JARID1A, JARID1B, JARID1C, JARID1D, and JHDM1B). Although functional redundancy may exist, knockout and knockdown experiments demonstrate that each enzyme performs distinctive cellular functions [26, 29, 31, 33, 55, 56]. The specific cellular function may be regulated by the spatial and temporal expression of the enzyme, the localization in specific chromosomal regions, or the binding to specific regulatory elements of a gene. It can also be regulated by other proteins in a context-dependent manner.

How KIAA1718 is recruited to the promoter region of FGF4 gene remains to be determined. It is possible that KIAA1718 is recruited by a protein complex that contains DNA- or histone-binding activities. Since KIAA1718 has a PHD domain, which binds methylated H3K4 and H3K9 [31, 57, 58], it is also possible that KIAA1718 is recruited by pre-existing epigenetic marks.

Since H3K9me2 and H3K27me2 are important epigenetic marks associated with transcription repression, a histone demethylase for these two marks may have many biological functions. Future studies using transgenic and knockout mouse models will reveal other biological functions of KIAA1718.

\section{Materials and Methods}

\section{Reagents}

Sources of the antibodies used are as follows: H3 monomethylK4 (Abcam 8895), H3 dimethy-K4 (Upstate 07-030), H3 trimethyl-K4 (Upstate 05-745), H3 monomethyl-K9 (Abcam 9045), H3 dimethyl-K9 (Upstate 07-441), H3 trimethyl-K9 (Abcam 8898), H3 monomethyl-K27 (Upstate 07-448), H3 dimethyl-K27 (Upstate 07-452 and Abcam 24684), H3 trimethyl-K27 (Upstate 07-449), H3 monomethyl-K36 (Abcam 9048), H3 dimethyl-K36 (Upstate 07-369), H3 trimethyl-K36 (Abcam 9050), H3 (abcam1791), Goat anti-Rabbit IgG and Goat anti-Mouse IgG (Jackson), Donkey anti-Rabbit (Molecular Probes Alexa 594) and Goat anti-Mouse (Molecular Probes Alexa 488), Anti-Flag M2 affinity gel (SigmaA2220). The chemicals are $\alpha$-ketoglutaric acid disodium salt dehydrate (Sigma Cat 75892), ascorbic acid (Sigma Cat A2218), ammonium iron (II) sulfate hexahydrate (Sigma Cat F1543), NiNTA agarose (25 ml) (Qiagen Cat 30210), KIAA1718 antibody (Abcam 36044). Antibodies against Oct4, Nestin, Tuj1 (neuronal class III $\beta$-tubulin), MAP2, and GFP were purchased from Santa Cruz Biotechnology (Santa Cruz, CA), R\&D Systems (Minneapolis, MN), BD Biosciences (San Jose, CA), Covance Research Products (Denver, PA), Sigma-Aldrich (St Louis, MO), and Invitrogen (Carlsbad, CA).

\section{Cloning procedures}

pFlag-KIAA1718-IRES2-EGFP was constructed by ligating the fragments of 1-160 bp by synthesis and 160-2 826 bp by RTPCR into pIRES2-EGFP and sequenced. The H281A mutant was generated by QuickChange (Stratagene) according to the manufacturers instructions. The full-length wild-type KIAA1718 cDNA and H281A mutant were digested from pFlag-KIAA1718-IRES2EGFP by NheI and XhoI, and ligated to $X b a \mathrm{I}$ and $X h o I$-digested $\mathrm{pFastBacT} 1$ (Invitrogen) vector to engineer insect expression vector pFastBacT1-his-KIAA1718 that contains a C-terminal $6 \times$ His for affinity purification. They were also subcloned into lentiviral vectors pFUGW-IRES-EGFP and pCSII-IRES-DsRed 2 for overexpression and rescue experiments, respectively. 
Recombinant KIAA1718 and H281A mutant

Tn5 cells were infected with baculovirus, collected $72 \mathrm{~h}$ later, incubated in $20 \mathrm{mM}$ Tris, $1 \% \mathrm{NP}-40$ with protease inhibitors in ice for $30 \mathrm{~min}$, and sonicated 25 times at $200 \mathrm{~W}$ (sonicated for $2 \mathrm{~s}$ and paused for $15 \mathrm{~s}$ ). After centrifugation, the supernatant was loaded onto Ni-NTA column and washed. The recombinant proteins were eluted by $250 \mathrm{mM}$ imidazole and determined using SDS-PAGE followed by Coomassie blue staining.

\section{Demethylation assay}

Bulk histones were incubated with the purified his-KIAA1718 in demethylation buffer (20 mM Tris- $\mathrm{HCl}(\mathrm{pH} 7.3), 150 \mathrm{mM} \mathrm{NaCl}$, $50 \mu \mathrm{M}\left(\mathrm{NH}_{4}\right)_{2} \mathrm{Fe}\left(\mathrm{SO}_{4}\right)_{2}+6\left(\mathrm{H}_{2} \mathrm{O}\right), 1 \mathrm{mM} \alpha$-ketoglutarate, and 2 $\mathrm{mM}$ ascorbic acid) for $3 \mathrm{~h}$ at $37{ }^{\circ} \mathrm{C}$. A total of 5-10 $\mu \mathrm{g}$ of the protein and 2.5-5 $\mu \mathrm{g}$ of bulk histones were reacted in a total volume of $100 \mu \mathrm{l}$ of reaction mixture. The reaction was stopped with SDS loading buffer and western blot analysis was performed.

\section{Chromatin immunoprecipitation}

Crosslinking was performed in $1 \%$ formaldehyde and sonication was carried out at $85 \mathrm{~Hz}$ for $10 \mathrm{~s}$ and paused for $30 \mathrm{~s}$ up to 75 cycles (Sonics Vibra Cell) to shear DNA to an average fragment size of 200-400 bp. Sonication buffer or FA lysis buffer was used for Flag or H3K9me2 IP, respectively. After de-crosslinking and protein digestion, DNA was precipitated and quantitative PCR was performed.

\section{Cell culture and neural differentiation}

The mouse ES cell line R1 was grown on a layer of mouse embryonic fibroblasts and in culture medium (DMEM supplemented with $15 \%$ fetal calf serum, $1000 \mathrm{U} / \mathrm{ml}$ leukemia inhibitory factor, L-glutamine, and non-essential amino acids). The ES cell neural differentiation was performed as previously described $[45,59]$.

\section{$R N A i$ and rescue procedures}

For RNA interference (RNAi) experiments, lentiviral vector pLentiLox 3.7 expressing shRNA was used for lentivirial packaging and lentiviral transduction of ESCs. The shRNA constructs (pLentiLox 3.7-shRNAs) targeting firefly luciferase (control) and three different KIAA1718 sequences were shown below:

Control shRNA, 5'-CTT GTG AAG CTT CTA GGA G-3'; KIAA1718 shRNA1, 5'-GAG CAT CAT GCT GTG GAC A-3'; KIAA1718 shRNA2, 5'-GTG CTA AAT GTG ATC AGC C-3'; and KIAA1718 shRNA3, 5'-TGA AGA AGA GGA GGA ACT G-3'.

After 48-h lentiviral transfection, GFP-positive ES cells were sorted using FACS Aria cell sorter (BD Biosciences). For rescue experiments, human wild-type KIAA1718 and H281A mutants were constructed into lentiviral vector pCSII-IRES-DsRed 2 for lentiviral packaging, and were used for co-transfection with the RNAi lentivirus into the ES cells. After 48-h transfection, GFP and RFP double-positive ES cells were sorted for analyses.

Gene overexpression in ES cells and time-course analysis

Human wild-type KIAA1718 and H281A mutant were constructed into lentiviral vector pFUGW-IRES-EGFP for overexpression in ES cells. After standard lentiviral packaging and lentiviral transduction of ES cells, the neural differentiation was performed. The embryonic bodies from three experimental groups, including the control, hKIAA1718, mut hKIAA1718, were fixed on every 2 days and subjected to immunostaining.

\section{Immunocytochemistry}

Embryoid bodies derived from ES cells were fixed in 4\% (wt/ vol) formaldehyde for $2 \mathrm{~h}$ at $4{ }^{\circ} \mathrm{C}$ and subjected to frozen section. The cell sections were permeabilized in $0.2 \%$ Triton X-100 PBS buffer and incubated with the indicated primary and secondary antibodies (see Regents section). Fluorescence detection and imaging

Table 1 Primer sequences for RT-PCR and Q-PCR

\begin{tabular}{|c|c|}
\hline$\overline{\text { Gapdh-F }}$ & 5'-TGACCACAGTCCATGCCATC-3' \\
\hline Gapdh-R & 5'-GACGGACACATTGGGGGTAG-3' \\
\hline mKIAA1718-F & 5'-GGAACTGGCACAGGCATGACTA-3' \\
\hline mKIAA1718-R & 5'-CCTGAGTCCAAGGTCATCTAATTTA-3' \\
\hline mFgf1-F & 5'-TTGTCAAGAGAGATGCTATGGATTC-3 \\
\hline mFgf1-R & 5'-CCTGTCTCTGTCACTTTGTTGGG-3' \\
\hline mFgf2-F & 5'-GGTCACGGAAATACTCCAGTTG-3' \\
\hline mFgf2-R & 5'-CTTAGCAGACATTGGAAGAAACAGT-3' \\
\hline mFgf3-F & 5'-CTGGAAGACTCGAAGTTCAAGATGT-3' \\
\hline mFgf3-R & 5'-GCCAGGACTGACTGCCATTG-3' \\
\hline mFgf4-F & 5'-TGTGGTCCTACCTGACACCTATTAC-3' \\
\hline mFgf4-R & 5'-CGGTAACAGTGGCAGATACAGAGC-3' \\
\hline mFgf7-F & 5'-CGAAGAAAGAACAAAAAACAGCC-3' \\
\hline $\mathrm{mFgf7}-\mathrm{R}$ & 5'-CTCCCTGCTGAATGAAACTGGT-3' \\
\hline mFgf13-F & 5'-GTCCTCCTGTCATTGGATTTGG-3' \\
\hline mFgf13-R & 5'-CAACTCTATGCCAAGATTCCAACT-3' \\
\hline mFgf15-F & 5'-ATCGGAGCCCCACTGACTTT-3' \\
\hline mFgf15-R & 5'-CATTTGACTTCATACCTTCTGGCTT-3' \\
\hline mFgf17-F & 5'-GTCTCCTTGACCTAAAATGCCTG-3' \\
\hline mFgf17-R & 5'-TTTGGCTATTTGGTTCAGATTCAG-3' \\
\hline mFgf18-F & 5'-TTCTGGAAAACAACTACACGGC-3' \\
\hline mFgf18-R & 5'-TGGTGACTGTGGTGTATTTGAAGG-3' \\
\hline $\mathrm{mFgf} 23-\mathrm{F}$ & 5'-GTTACTATTCTGGGATTCACGGGT-3' \\
\hline $\mathrm{mFgf} 23-\mathrm{R}$ & 5'-ACTCTGAAATGTGTTCGGTTTAGGT-3' \\
\hline mOct4-F & 5'-AGTTGGCGTGGAGACTTTGC-3' \\
\hline mOct4-R & 5'-CAGGGCTTTCATGTCCTGG-3' \\
\hline mBrachyury-F & 5'-CTCGGATTCACATCGTGAGAG-3' \\
\hline mBrachyury-R & 5'-AAGGCTTTAGCAAATGGGTTGTA-3' \\
\hline $\mathrm{mFlk1-F}$ & 5'-GGGTCGATTTCAAACCTCAATGT-3' \\
\hline mFlk1-R & 5'-AGAGTAAAGCCTATCTCGCTGT-3' \\
\hline mGATA4-F & 5'-CCCTACCCAGCCTACATGG-3' \\
\hline mGATA4-R & 5'-ACATATCGAGATTGGGGTGTCT-3' \\
\hline mGATA6-F & 5'-TTGCTCCGGTAACAGCAGTG-3' \\
\hline mGATA6-R & 5'-GTGGTCGCTTGTGTAGAAGGA-3' \\
\hline mRex 1-F & 5'-CCCTCGACAGACTGACCCTAA-3' \\
\hline mRex1-R & 5'-TCGGGGCTAATCTCACTTTCAT-3' \\
\hline mNanog-F & 5'-TCTTCCTGGTCCCCACAGTTT-3' \\
\hline mNanog-R & 5'-GCAAGAATAGTTCTCGGGATGAA-3' \\
\hline
\end{tabular}


were carried out on Leica SP5 confocal microscope.

\section{Microarray and RT-PCR analysis}

Total RNA from ES cells was isolated using RNeasy Total RNA Isolation Kit from QIAGEN. GeneChip expression analysis was performed according to the Affymetrix standard instruction. Mouse Genome 4302.0 microarrays were used for gene expression screening in this study. Primer sequences for RT-PCR and QPCR used in this study are showed in Table 1.

\section{Acknowledgments}

We thank Anning Lin (The University of Chicago) for the critical reading of the paper, members in the Chen lab for technical help, the cell biology and molecular biology core facilities for confocal study and Q-PCR, and Shanghai Biochip Co Ltd. for microarray analysis. The H3K27me2 antibody was kindly provided by Li Tang (Fudan University) and Thomas Jenuwein (Research Institute of Molecular Pathology, The Vienna Biocenter). This work was supported by the National Basic Research Program of China (2007CB957900, 2006CB943902, 2007CB947101, 2008KR0695, 2009CB941100, 2005CB522704), the Chinese Academy of Sciences (KSCX2-YW-R-04), the National Natural Science Foundation of China (90919026, 30870538,30623003, 30721065, 30830034, 90919046), the Shanghai Pujiang Program (0757S11361), the Shanghai Key Project of Basic Science Research (06DJ14001, 06DZ22032, 08DJ1400501), and the Council of Shanghai Municipal Government for Science and Technology (088014199).

\section{References}

1 Luger K, Mader AW, Richmond RK, Sargent DF, Richmond TJ. Crystal structure of the nucleosome core particle at $2.8 \AA$ resolution. Nature 1997; 389:251-260.

2 Zhang Y, Reinberg D. Transcription regulation by histone methylation: interplay between different covalent modifications of the core histone tails. Genes Dev 2001; 15:23432360.

3 Bhaumik SR, Smith E, Shilatifard A. Covalent modifications of histones during development and disease pathogenesis. Nat Struct Mol Biol 2007; 14:1008-1016.

4 Strahl BD, Allis CD. The language of covalent histone modifications. Nature 2000; 403:41-45.

5 Peters AH, Kubicek S, Mechtler K, et al. Partitioning and plasticity of repressive histone methylation states in mammalian chromatin. Mol Cell 2003; 12:1577-1589.

6 Rice JC, Briggs SD, Ueberheide B, et al. Histone methyltransferases direct different degrees of methylation to define distinct chromatin domains. Mol Cell 2003; 12:1591-1598.

7 Tachibana M, Sugimoto K, Fukushima T, Shinkai Y. Set domain-containing protein, G9a, is a novel lysine-preferring mammalian histone methyltransferase with hyperactivity and specific selectivity to lysines 9 and 27 of histone H3. J Biol Chem 2001; 276:25309-25317.

8 Tachibana M, Sugimoto K, Nozaki M, et al. G9a histone methyltransferase plays a dominant role in euchromatic histone H3 lysine 9 methylation and is essential for early embryogenesis. Genes Dev 2002; 16:1779-1791.
9 Schultz DC, Ayyanathan K, Negorev D, Maul GG, Rauscher FJ, 3rd. SETDB1: a novel KAP-1-associated histone H3, lysine 9-specific methyltransferase that contributes to HP1mediated silencing of euchromatic genes by KRAB zincfinger proteins. Genes Dev 2002; 16:919-932.

10 Tachibana M, Ueda J, Fukuda M, et al. Histone methyltransferases G9a and GLP form heteromeric complexes and are both crucial for methylation of euchromatin at H3-K9. Genes Dev 2005; 19:815-826.

11 Wang H, An W, Cao R, et al. mAM facilitates conversion by ESET of dimethyl to trimethyl lysine 9 of histone $\mathrm{H} 3$ to cause transcriptional repression. Mol Cell 2003; 12:475-487.

12 Yang L, Xia L, Wu DY, et al. Molecular cloning of ESET, a novel histone $\mathrm{H} 3$-specific methyltransferase that interacts with ERG transcription factor. Oncogene 2002; 21:148-152.

13 Dodge JE, Kang YK, Beppu H, Lei H, Li E. Histone H3-K9 methyltransferase ESET is essential for early development. Mol Cell Biol 2004; 24:2478-2486.

14 Barski A, Cuddapah S, Cui K, et al. High-resolution profiling of histone methylations in the human genome. Cell 2007; 129:823-837.

15 Cao R, Wang L, Wang H, et al. Role of histone H3 lysine 27 methylation in Polycomb-group silencing. Science 2002; 298:1039-1043.

16 Kuzmichev A, Nishioka K, Erdjument-Bromage H, Tempst $\mathrm{P}$, Reinberg D. Histone methyltransferase activity associated with a human multiprotein complex containing the Enhancer of Zeste protein. Genes Dev 2002; 16:2893-2905.

17 Faust C, Lawson KA, Schork NJ, Thiel B, Magnuson T. The Polycomb-group gene eed is required for normal morphogenetic movements during gastrulation in the mouse embryo. Development 1998; 125:4495-4506.

18 O'Carroll D, Erhardt S, Pagani M, et al. The polycomb-group gene Ezh2 is required for early mouse development. Mol Cell Biol 2001; 21:4330-4336.

19 Pasini D, Bracken AP, Jensen MR, Lazzerini Denchi E, Helin K. Suz12 is essential for mouse development and for EZH2 histone methyltransferase activity. EMBO J 2004; 23:40614071.

20 Shi Y, Lan F, Matson C, et al. Histone demethylation mediated by the nuclear amine oxidase homolog LSD1. Cell 2004; 119:941-953.

21 Tsukada Y, Fang J, Erdjument-Bromage H, et al. Histone demethylation by a family of JmjC domain-containing proteins. Nature 2006; 439:811-816.

22 Klose RJ, Kallin EM, Zhang Y. JmjC-domain-containing proteins and histone demethylation. Nat Rev Genet 2006; 7:715727.

23 Shi Y, Whetstine JR. Dynamic regulation of histone lysine methylation by demethylases. Mol Cell 2007; 25:1-14.

24 Xiang Y, Zhu Z, Han G, et al. JARID1B is a histone H3 lysine 4 demethylase up-regulated in prostate cancer. Proc Natl Acad Sci USA 2007; 104:19226-19231.

25 Lee MG, Villa R, Trojer P, et al. Demethylation of H3K27 regulates polycomb recruitment and $\mathrm{H} 2 \mathrm{~A}$ ubiquitination. Science 2007; 318:447-450.

26 Lee MG, Norman J, Shilatifard A, Shiekhattar R. Physical and functional association of a trimethyl $\mathrm{H} 3 \mathrm{~K} 4$ demethylase and Ring6a/MBLR, a polycomb-like protein. Cell 2007; 128:877- 
887.

27 Xiang Y, Zhu Z, Han G, et al. JMJD3 is a histone H3K27 demethylase. Cell Res 2007; 17:850-857.

28 Lan F, Bayliss PE, Rinn JL, et al. A histone H3 lysine 27 demethylase regulates animal posterior development. Nature 2007; 449:689-694.

29 Christensen J, Agger K, Cloos PA, et al. RBP2 belongs to a family of demethylases, specific for tri- and dimethylated lysine 4 on histone 3. Cell 2007; 128:1063-1076.

30 Cloos PA, Christensen J, Agger K, et al. The putative oncogene GASC1 demethylates tri- and dimethylated lysine 9 on histone H3. Nature 2006; 442:307-311.

31 Iwase S, Lan F, Bayliss P, et al. The X-linked mental retardation gene SMCX/JARID1C defines a family of histone H3 lysine 4 demethylases. Cell 2007; 128:1077-1088.

32 Klose RJ, Yamane K, Bae Y, et al. The transcriptional repressor JHDM3A demethylates trimethyl histone H3 lysine 9 and lysine 36. Nature 2006; 442:312-316.

33 Klose RJ, Yan Q, Tothova Z, et al. The retinoblastoma binding protein RBP2 is an H3K4 demethylase. Cell 2007; 128:889900.

34 Whetstine JR, Nottke A, Lan F, et al. Reversal of histone lysine trimethylation by the JMJD2 family of histone demethylases. Cell 2006; 125:467-481.

35 Yamane K, Tateishi K, Klose RJ, et al. PLU-1 is an H3K4 demethylase involved in transcriptional repression and breast cancer cell proliferation. Mol Cell 2007; 25:801-812.

36 Yamane K, Toumazou C, Tsukada Y, et al. JHDM2A, a JmjCcontaining $\mathrm{H} 3 \mathrm{~K} 9$ demethylase, facilitates transcription activation by androgen receptor. Cell 2006; 125:483-495.

37 Agger K, Cloos PA, Christensen J, et al. UTX and JMJD3 are histone H3K27 demethylases involved in HOX gene regulation and development. Nature 2007; 449:731-734.

38 Stern CD. Neural induction: old problem, new findings, yet more questions. Development 2005; 132:2007-2021.

39 Wilson SI, Graziano E, Harland R, Jessell TM, Edlund T. An early requirement for FGF signalling in the acquisition of neural cell fate in the chick embryo. Curr Biol 2000; 10:421429.

40 Kunath T, Saba-El-Leil MK, Almousailleakh M, et al. FGF stimulation of the Erk1/2 signalling cascade triggers transition of pluripotent embryonic stem cells from self-renewal to lineage commitment. Development 2007; 134:2895-2902.

41 Tropepe V, Hitoshi S, Sirard C, et al. Direct neural fate specification from embryonic stem cells: a primitive mammalian neural stem cell stage acquired through a default mechanism. Neuron 2001; 30:65-78.

42 Stavridis MP, Lunn JS, Collins BJ, Storey KG. A discrete period of FGF-induced Erk1/2 signalling is required for vertebrate neural specification. Development 2007; 134:28892894.

43 LaVaute TM, Yoo YD, Pankratz MT, et al. Regulation of neu- ral specification from human embryonic stem cells by BMP and FGF. Stem Cells 2009; 27:1741-1749.

44 Finley MF, Devata S, Huettner JE. BMP-4 inhibits neural differentiation of murine embryonic stem cells. $J$ Neurobiol 1999; 40:271-287.

45 Kawasaki H, Mizuseki K, Nishikawa S, et al. Induction of midbrain dopaminergic neurons from ES cells by stromal cell-derived inducing activity. Neuron 2000; 28:31-40.

46 Allis CD, Berger SL, Cote J, et al. New nomenclature for chromatin-modifying enzymes. Cell 2007; 131:633-636.

47 Chen Z, Zang J, Kappler J, et al. Structural basis of the recognition of a methylated histone tail by JMJD2A. Proc Natl Acad Sci USA 2007; 104:10818-10823.

48 Chen Z, Zang J, Whetstine J, et al. Structural insights into histone demethylation by JMJD2 family members. Cell 2006; 125:691-702.

49 Couture JF, Collazo E, Ortiz-Tello PA, Brunzelle JS, Trievel RC. Specificity and mechanism of JMJD2A, a trimethyllysine-specific histone demethylase. Nat Struct Mol Biol 2007; 14:689-695.

$50 \mathrm{Ng} \mathrm{SS}$, Kavanagh KL, McDonough MA, et al. Crystal structures of histone demethylase JMJD2A reveal basis for substrate specificity. Nature 2007; 448:87-91.

51 Frescas D, Guardavaccaro D, Bassermann F, Koyama-Nasu R, Pagano M. JHDM1B/FBXL10 is a nucleolar protein that represses transcription of ribosomal RNA genes. Nature 2007; 450:309-313.

52 He J, Kallin EM, Tsukada Y, Zhang Y. The H3K36 demethylase $\mathrm{Jhdm} 1 \mathrm{~b} / \mathrm{Kdm} 2 \mathrm{~b}$ regulates cell proliferation and senescence through p15(Ink4b). Nat Struct Mol Biol 2008; 15:1169-1175.

53 Agger K, Christensen J, Cloos PA, Helin K. The emerging functions of histone demethylases. Curr Opin Genet Dev 2008; 18:159-168.

54 Shi Y. Histone lysine demethylases: emerging roles in development, physiology and disease. Nat Rev Genet 2007; 8:829833.

55 Wang J, Hevi S, Kurash JK, et al. The lysine demethylase LSD1 (KDM1) is required for maintenance of global DNA methylation. Nat Genet 2009; 41:125-129.

56 Wang J, Scully K, Zhu X, et al. Opposing LSD1 complexes function in developmental gene activation and repression programmes. Nature 2007; 446:882-887.

57 Shi X, Hong T, Walter KL, et al. ING2 PHD domain links histone $\mathrm{H} 3$ lysine 4 methylation to active gene repression. Nature 2006; 442:96-99.

58 Wysocka J, Swigut T, Xiao H, et al. A PHD finger of NURF couples histone $\mathrm{H} 3$ lysine 4 trimethylation with chromatin remodelling. Nature 2006; 442:86-90.

59 Watanabe K, Kamiya D, Nishiyama A, et al. Directed differentiation of telencephalic precursors from embryonic stem cells. Nat Neurosci 2005; 8:288-296.

(Supplementary information is linked to the online version of the paper on the Cell Research website.) 